\title{
ARTICLE \\ Abnormalities in electroencephalographic microstates are state and trait markers of major depressive disorder
}

\author{
Michael Murphy $\mathbb{D}^{1,2}$, Alexis E. Whitton ${ }^{10,2,4}$, Stephanie Deccy ${ }^{2,5}$, Manon L. Ironside ${ }^{2,6}$, Ashleigh Rutherford ${ }^{2,7}$, Miranda Beltzer ${ }^{2,8}$, \\ Matthew Sacchet ${ }^{1,2}$ and Diego A. Pizzagalli (iD)
}

\begin{abstract}
Neuroimaging studies have shown that major depressive disorder (MDD) is characterized by abnormal neural activity and connectivity. However, hemodynamic imaging techniques lack the temporal resolution needed to resolve the dynamics of brain mechanisms underlying MDD. Moreover, it is unclear whether putative abnormalities persist after remission. To address these gaps, we used microstate analysis to study resting-state brain activity in major depressive disorder (MDD). Electroencephalographic (EEG) "microstates" are canonical voltage topographies that reflect brief activations of components of resting-state brain networks. We used polarity-insensitive k-means clustering to segment resting-state high-density (128-channel) EEG data into microstates. Data from 79 healthy controls (HC), 63 individuals with MDD, and 30 individuals with remitted MDD (rMDD) were included. The groups produced similar sets of five microstates, including four widely-reported canonical microstates (A-D). The proportion of microstate D was decreased in MDD and rMDD compared to the HC group (Cohen's $d=0.63$ and 0.72 , respectively) and the duration and occurrence of microstate D was reduced in the MDD group compared to the HC group (Cohen's $d=0.43$ and 0.58 , respectively). Among the MDD group, proportion and duration of microstate D were negatively correlated with symptom severity (Spearman's rho $=-0.34$ and -0.46 , respectively). Finally, microstate transition probabilities were nonrandom and the MDD group, relative to the $\mathrm{HC}$ and the rMDD groups, exhibited multiple distinct transition probabilities, primarily involving microstates $\mathrm{A}$ and $\mathrm{C}$. Our findings highlight both state and trait abnormalities in resting-state brain activity in MDD.
\end{abstract}

Neuropsychopharmacology (2020) 45:2030-2037; https://doi.org/10.1038/s41386-020-0749-1

\section{INTRODUCTION}

Major depressive disorder (MDD) is a prevalent psychiatric illness and major source of disability [1]. Converging evidence suggests that MDD is a disconnection syndrome [2]. According to this model, psychiatric symptoms are related to disconnection between brain regions and effective treatments may act by normalizing or compensating for this dysconnectivity. Fitting this, functional magnetic resonance imaging (fMRI) and electroencephalography (EEG) findings implicate abnormal functioning in MDD within and between multiple resting-state brain networks $[2,3]$. Therefore, in order to better elucidate the pathophysiology of MDD, it is important to advance our understanding of dynamics of large-scale brain networks.

EEG can be used to study the coordinated action of large populations of neurons with exquisite temporal resolution. Crucially, resting-state EEG is dominated by a small number of characteristic semi-stable voltage topographies [4, 5]. During an EEG recording, a specific topography (or "microstate") will persist for tens of microseconds, and then transition to a different topography. This observation led to the development of microstate analysis, which is the identification and quantification of these topographies [6]. Advancements in clustering algorithms and computing power have allowed for more precise study of microstates, and multiple studies have confirmed the basic principle that a large amount of the EEG signal can be parsimoniously explained by a small number of characteristic microstates $[7,8]$. Microstates are consistent across studies, with a canonical set of four microstates "A-D" being identified in most reports [7]. Microstates are generated by distinct, but overlapping, neural assemblies and their parameters and topographical transitions provide information about large-scale brain connectivity [8-10]. Taken together, microstate analysis is a powerful datadriven approach for functional mapping of large-scale brain networks.

While microstate analysis has been widely used to study brain function, few studies have probed microstates in affective disorders [11]. This literature is equivocal, with reports of inconsistent abnormalities in microstate duration and transition probabilities [12-14]. Furthermore, previous studies have used diagnostically mixed populations, aggregating individuals with

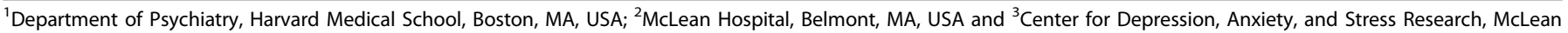
Hospital, Belmont, MA, USA

Correspondence: Diego A. Pizzagalli (dap@mclean.harvard.edu)

${ }^{4}$ Present address: University of Sydney, Sydney, Australia

${ }^{5}$ Present address: Albert Einstein College of Medicine, Bronx, NY, USA

${ }^{6}$ Present address: University of California-Berkeley, Berkeley, CA, USA

${ }^{7}$ Present address: Yale University, New Haven, CT, USA

${ }^{8}$ Present address: University of Virginia, Charlottesville, VA, USA
}

Received: 11 February 2020 Revised: 15 June 2020 Accepted: 17 June 2020

Published online: 26 June 2020 
Table 1. Demographic and clinical data.

\begin{tabular}{llll}
\hline & Controls & MDD & rMDD \\
\hline Sample size & 79 & 63 & 30 \\
Age in years, mean (SEM) & $27.5(0.9)$ & $29.2(1.1)$ & $32.7(2.7)$ \\
Sex, $\mathrm{N}_{\text {male }} / \mathrm{N}_{\text {female }}$ & $21 / 58$ & $21 / 42$ & $8 / 22$ \\
Education, years (SEM) & $16.5(0.3)$ & $16.0(0.3)$ & $16.5(0.4)$ \\
Current psychotropic medication, $\mathrm{N}$ & 0 & 10 & 0 \\
BDI-II Score, mean (SEM) & $0.7(0.2)$ & $26.7(1.2)$ & $2.8(0.6)$ \\
\hline
\end{tabular}

$H C$ healthy control, $M D D$ major depressive disorder, $r M D D$ remitted major depressive disorder, SEM standard error of the mean, $B D I-I /$ Beck Depression Inventory-ll.

unipolar and bipolar depression [14]. This is problematic because unipolar and bipolar depression respond to different treatments suggesting different pathophysiologies. Here, we critically expand on past research by performing the largest microstate study entirely of patients with MDD. A group with remitted MDD (rMDD) was also included, allowing us to identify putative state vs. trait microstate markers of depression. We hypothesized that microstate dynamics would differ between patients with MDD and healthy controls and that these differences would be partially attenuated in patients with rMDD.

\section{METHODS}

Participants and procedure

This work complies with the ethical standards of the relevant national and institutional committees on human experimentation and with the Helsinki Declaration of 1975, as revised in 2008. All procedures were approved by the Institutional Review Board of Partners Healthcare. Participants provided written informed consent for all study procedures. Data from 65 patients with MDD, 30 individuals with rMDD, and 79 age-matched healthy controls (HC) were available for analyses and were collected as part of a prior study [3]. Twenty-nine patients with MDD were treatment-seeking, 10 were taking psychotropic medication. We excluded individuals with a history of neurological disorder, seizures, and head injury. Participants were required to have a negative urine drug screen. MDD participants were required to have an MDD diagnosis according to the Structured Clinical Interview for DSM-IV (SCID) and to have had no changes to their psychiatric medications in the past eight weeks or have taken no medication for two weeks (six for venlafaxine). RMDD participants were required to have had a major depressive episode within the past five years, to be in remission for the past eight weeks (assessed with the SCID), and not be taking psychotropic medications [15]. Control participants had no history of psychiatric illness, no first-degree relatives with psychiatric disorders, and no history of psychiatric medication use. All participants were administered the SCID to establish diagnosis and the Beck Depression Inventory II (BDI-II) to assess depressive severity [16]. Subject characteristics are summarized in Table 1. For one MDD participant, BDI-II, medication, and education data were not available.

\section{Electroencephalography}

EEG data were collected in an electrically and acoustically shielded room using a 129-channel $\mathrm{Ag}-\mathrm{AgCl}$ electrode Hydrocel Geodesic Sensor Net (Electrical Geodesics; Koninklijke Philips, Amsterdam, The Netherlands). Data were collected at a $250 \mathrm{~Hz}$ sampling rate, vertex-referenced, with impedances $<100 \mathrm{k} \Omega$. Eight minutes of resting-state data were recorded in one-minute segments, four segments with eyes open and four with eyes closed. The order of recording was counterbalanced across participants. Consistent with past work, only eyes closed data were analyzed [17]. Data were exported to BrainVision Analyzer 2.0 (Brain Products $\mathrm{GmbH}$, Gilching, Germany). Artifactual segments were identified by visual inspection and removed. Independent component analysis was used to remove eye blinks and electrocardiogram artifact [18]. Corrupted channels were visually identified and spline interpolated [19]. Data were then re-referenced to the average reference. Two MDD participants did not have sufficient data for microstate analyses and were excluded. The final MDD group was thus comprised of 63 individuals.

\section{Microstate analysis}

Microstate analysis was performed using CARTOOL (brainmapping.unige.ch/cartool, Denis Brunet). For each participant, we calculated the global mean field power (GFP) across all electrodes. For microstate segmentation, we downsampled the data by only including points where the GFP had a local maximum. Microstate transitions preferentially occur at GFP local minima and this downsampling does not significantly impact microstate segmentation results $[20,21]$. The data were then spatially filtered to enhance signal-to-noise ratio [22]. Next, the data were run through a series of polarity-insensitive k-means clustering analyses with $k$ ranging from 1 to 12 . A meta-criterion based on taking the median value of six distinct functions was used to determine the optimal number of microstates [23-26]. We subsequently ran a second round of clustering on the pooled microstates for subjects within the control, MDD, and rMDD groups. The meta-criterion was used to determine the optimal number of microstates. For microstate fitting, for each time point in the original data, we chose the microstate map from the subject's group that most closely resembled the voltage topography insensitive to polarity [21]. Fits were smoothed on 3 sample half-windows with Besag factor 10 [27]. We obtained a sequence of microstate labels with the same length as the original data set. For each record and microstate, we calculated three parameters. Proportion was the amount of the record covered by each microstate, duration was the average length of time a microstate lasted, and occurrence was the number of times that microstate occurred per second. For the fitted microstate sequences, we calculated a Markov matrix on the observed transitions between microstates [28]. We also calculated an expected Markov matrix by computing the expected transition probabilities given the distribution of microstate labels.

\section{Source modeling}

Source modeling was conducted using the Brainstorm toolbox in MATLAB (The MathWorks, Inc., Natick, MA) [29]. A standardized set of electrode locations was co-registered to the ICBM152 template head [30]. This was used to create a boundary-element head model which models propagation of electrical current from a set of cortical voxels through the head to the EEG channels. Unconstrained, standardized low-resolution electromagnetic tomography (sLORETA) with Tikhonov regularization, was used to normalize the minimum norm estimate and model cortical currents underlying the EEG microstate topographies [31]. Ideally, source modeling would be performed on all the EEG data and grouped by microstate label as the microstate maps are highly smoothed artificial topographies [26]. Given the large amount of EEG data, this was computationally intractable and therefore we performed source modeling on the microstate template maps. In order to compare localizations across microstates, the cortical topographies associated with each microstate were normalized by the maximum current across voxels. Then, each voxel was assigned to the microstate label for which it had the largest normalized current value.

\section{Statistics}

Statistical analyses were performed using MATLAB. To evaluate group differences in microstate parameters (proportion, duration, 


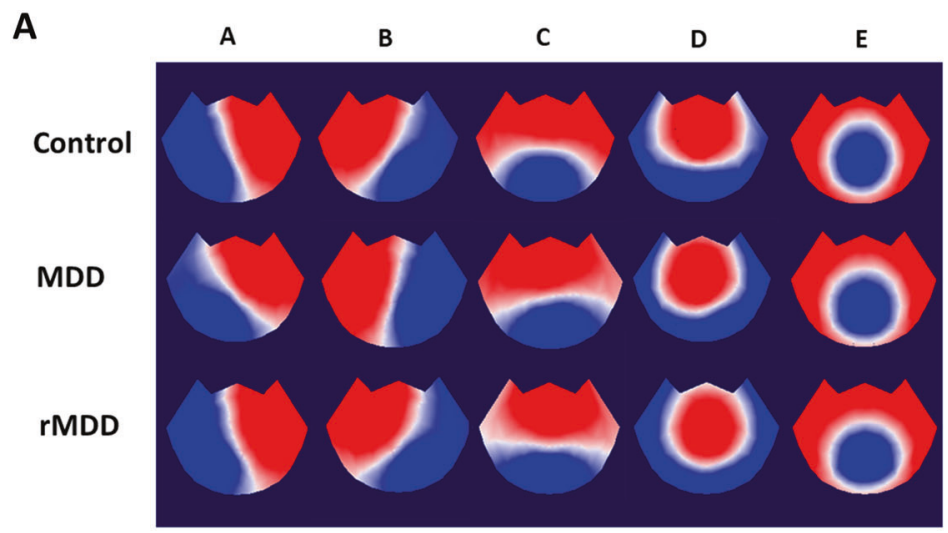

B

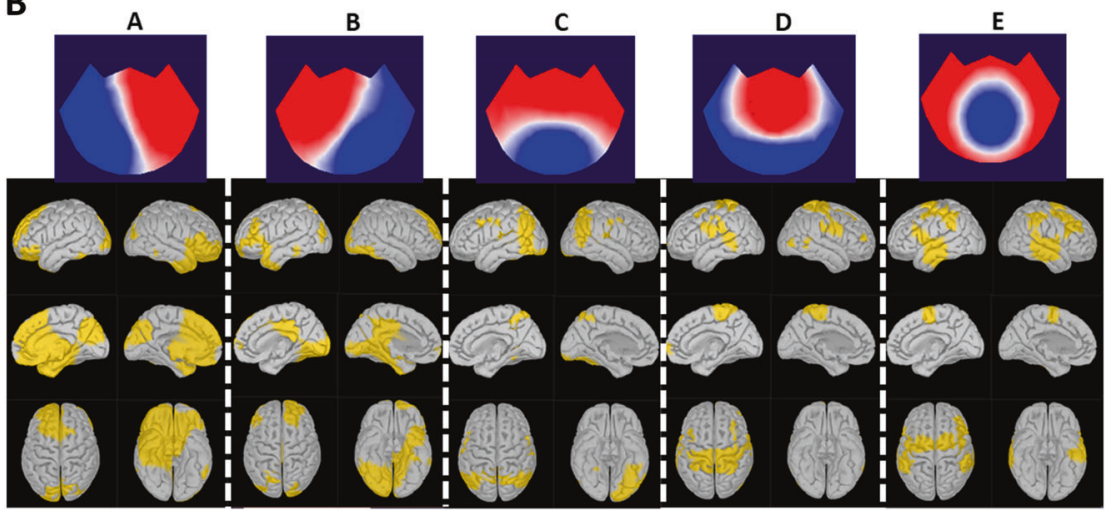

Fig. 1 All three groups produced similar sets of microstates which involve distinct but overlapping, cortical correlates. a EEG topoplots of microstate topographies obtained independently from the HC, MDD, and rMDD groups. Blue regions are negative and red are positive relative to average reference. Note that microstates are polarity invariant. These microstates are qualitatively similar across groups and include the canonical microstates A-D. b Top row shows the EEG topoplots for the microstates identified in the HC group. Below each microstate is a set of cortical source-localized maps indicating which voxels (yellow) were most strongly associated with the particular microstate.

and occurrence), we ran one-way ANOVAs and then used the Bonferroni correction for five comparisons (i.e., microstates) to adjust $\mathrm{p}$-values. Post-hoc testing was performed using the Tukey test (HSD). For microstates revealing group differences, Spearman correlation were used to analyze associations between microstate parameters and symptom scores and Wilcoxon tests were computed to evaluate effects of medications status.

\section{RESULTS}

Control, MDD, and rMDD groups produce similar sets of microstates

Microstate segmentation of resting-state EEG data from the $\mathrm{HC}$, MDD, and rMDD groups produced five microstate topographies (Fig. 1a) that were consistent across groups. The magnitude of the spatial correlation of the topographies between groups ranged from 0.889 (Map D, HC vs MDD) to 0.997 (Map A, HC vs rMDD). Previous studies have reported four canonical microstate topographies and these topographies are reflected in microstates A-D in the current data [11,32]. Moreover, some groups have reported additional microstates which resemble the current microstate $\mathrm{E}$ $[14,26]$. Microstate fits were calculated by measuring the correlation between the topography at each time point and its assigned microstate topography. The fits were equal across the three groups with average correlation coefficients and standard errors (SEM) of 0.699 (0.003) for the HC, 0.701 (0.003) for the MDD, and $0.700(0.005)$ for the rMDD group. Within the MDD group, there was no statistically significant effect of medication on average correlation coefficient (unpaired t-test, $\mathrm{t}(59)=0.31, p=$ 0.76). The global explained variance (GEV) of the models were also equal across groups with 0.606 (SEM 0.006) for the HC, 0.613 (SEM 0.006) for the MDD and 0.600 (SEM 0.012) for the rMDD.

Previous research suggests that patterns of neural activity that contribute to EEG microstates overlap with components of multiple resting-state networks [8]. To determine the cortical correlates of our microstates, the SLORETA algorithm was used to model the cortical currents associated with microstate topographies. To isolate how the cortical generators of microstates differed from each other, we focused our analysis on brain regions that were most strongly associated with each microstate and therefore possibly activated upon transition to that microstate (Fig. 1b). These regions were not uniquely activated during their associated microstate, but were most strongly associated with that microstate. These regions could be contiguous as in microstate $C$, which mostly involves the posteromedial cortex, or discontinuous as in microstate $A$, which involves the coordinated action of separate frontal and posterior cortical regions. Our microstates do not map precisely onto fMRI resting-state networks. Rather, microstate $A$ appears to involve anterior cingulate cortex, left insula, and occipital cortex; microstate $B$ involves posterior cingulate, right insula, and temporal cortex; microstate $C$ involves precuneus, postcentral cortex, and occipital cortex; microstate $D$ involves parietal and left insular cortex; and, finally, microstate E involves temporal, insular, and parietal cortices. Like the EEG topographies, the source models for the microstate templates were highly consistent across groups (spatial correlation across groups $>$.979). We note that although this method of source modeling the microstate templates may introduce bias, our source modeling results are consistent with previous work [26]. 
A
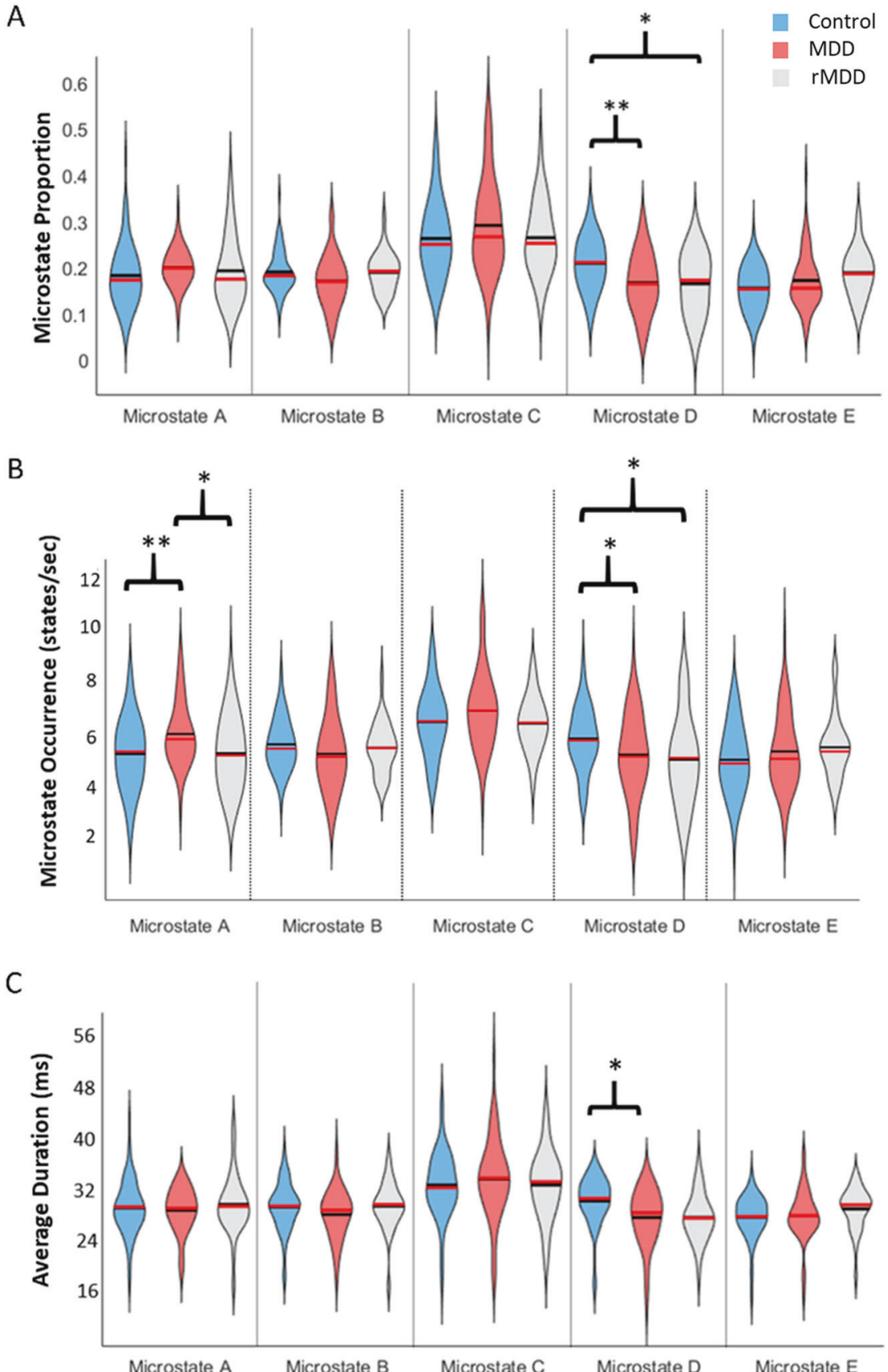

Fig. 2 Microstate D is decreased in MDD. a Violin plots showing the average proportion of each microstate in each of the three groups. Red lines are medians, black lines are means. Blue is the HC group, red is MDD, and gray is rMDD. $\mathbf{b}$ Violin plots showing average occurrence for each microstate in each of the three groups. c Violin plots showing average duration for each microstate in each of the three groups. ${ }^{*} p<0.05$. ${ }^{* *} p<0.01$ Tukey's HSD test following one-way following one-way ANOVA.

Microstate proportion, duration, and occurrence are altered in MDD and rMDD

Features of the fitted microstate sequences were compared across groups. We calculated the proportion of each EEG recording that was best fitted by each of the five microstates (Fig. 2a). Then, for each microstate, we calculated a one-way ANOVA comparing microstate proportions among groups. After correction for five multiple comparisons, we found evidence for a group effect for microstate $D\left(F_{2,171}=9.49, p_{\text {adjusted }}=0.0006\right)$. Post hoc testing (Tukey's HSD) clarified that microstate D covered a greater proportion of the recording in the HC $(0.208$, SEM 0.006) than MDD $(0.170$, SEM $0.006, p=0.002$, Cohen's $d=0.63$ ) or rMDD group (0.165, SEM 0.011, $p=0.0005$, Cohen's $d=0.72$ ). There were no statistically significant effects of age or years of education (all $p$ $>0.05$ ).

Next, we compared the occurrence of microstates in the three groups (Fig. 2b). ANOVA of microstate occurrence showed evidence for a group effect for microstate $D\left(F_{2,171}=4.81 p=\right.$ 0.008). Post-hoc tests clarified that microstate $D$ had higher average occurrence in the HC group (5.72, SEM 0.13 states/sec) compared to the MDD (5.11, SEM 0.20 states/sec, Tukey's HSD, $p=0.031$, Cohen's $d=0.44)$ and rMDD (4.92, SEM 0.30 states/sec, Tukey's HSD, $p=0.024$, Cohen's $d=0.56$ ) groups while there were no differences between MDD and rMDD. Post-hoc tests also showed that microstate A occurred more frequently in MDD (5.88, SEM 0.15 states/sec) than in HC (5.14, SEM 0.15 states/sec, Tukey's 

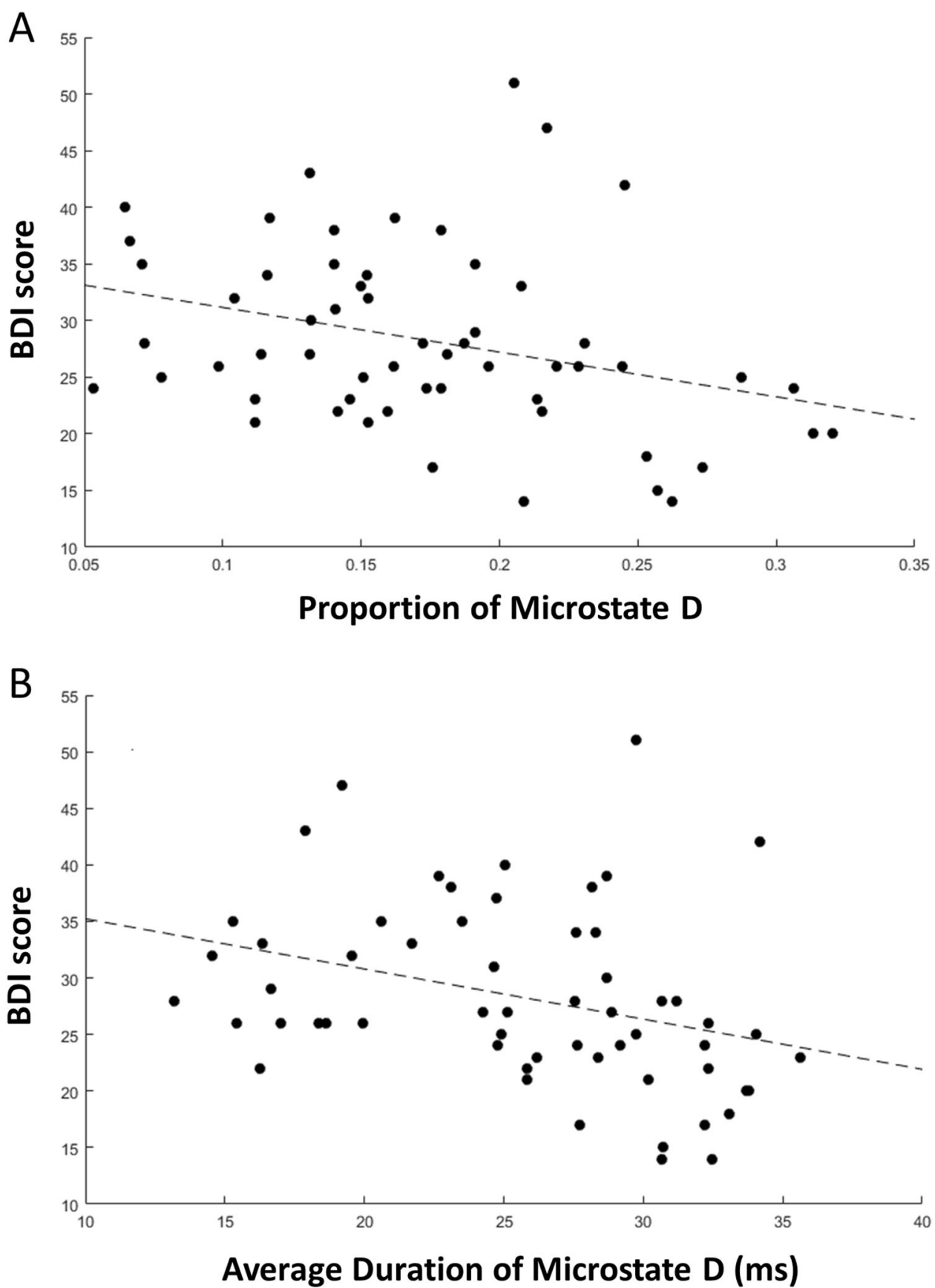

Fig. 3 Microstate $D$ is negatively correlated with depression symptoms. a Scatterplot of proportion of microstate $D$ and Beck Depression Index-II (BDI) total scores in patients with at least mild MDD (BDI $>9$ ). Spearman's rho $=-0.34, p=0.016$. b Scatterplot of duration of microstate D vs BDI scores in patients with at least mild MDD. Spearman's rho $=-0.46, p=0.0013$.

$\mathrm{HSD}, p=0.002$, Cohen's $d=0.59)$ or rMDD (5.16, SEM 0.23 states/ sec, Tukey's HSD, $\mathrm{p}=0.03$, Cohen's $d=0.58$ ).

We then compared the average durations of microstates in the three groups (Fig. 2c). For microstate D, the ANOVA showed a main effect of group $\left(F_{2,171}=3.99 p=0.02\right)$. Post-hoc tests clarified that microstate $\mathrm{D}$ had lower average duration in the MDD (25.54, SEM 0.75 ms) compared to HC group (28.01, SEM 0.60 ms, Tukey's HSD, $p=0.021$, Cohen's $d=0.43$ ). The rMDD (25.86, SEM $0.94 \mathrm{~ms}$ ) group did not differ from the HC (Tukey's HSD, $p=$ $0.16)$ or the MDD group (Tukey's HSD, $p=0.96$ ).

Finally, we examined the relationship between microstate parameters for microstates $A$ and $D$ and depression symptoms (Fig. 3). In the MDD group, 61 of the 63 individuals had BDI-II scores greater than 9, indicating at least mild depression. Within this group, we found that microstate $D$ proportion and duration were negatively associated with BDI-II scores (Spearman's rho = $-0.34, p=0.009$ and Spearman's rho $=-0.46, p=0.0002$, respectively). No statistically significant relationship emerged between microstate A or D occurrence and BDI-II scores (Spearman's rho = $0.13, p=0.33$ and Spearman's rho $=-0.16, p=0.22$, respectively). Within the MDD group, there was no statistically significant relationship between medication status and microstate $D$ proportion, duration, or occurrence (unpaired t-tests, $\mathrm{t}(59)=0.08, p=$ $0.94 ; \mathrm{t}(59)=-0.53, p=0.60 ; \mathrm{t}(59)=0.28, p=0.78$ ), or between medication status and symptom scores (Wilcoxon rank sum test, $p=0.65)$. 
A

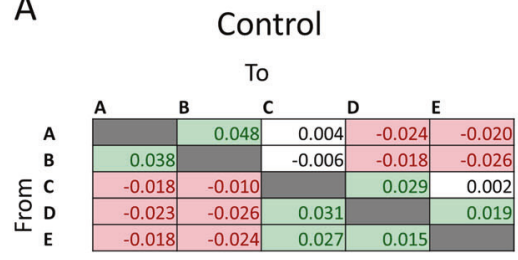

B

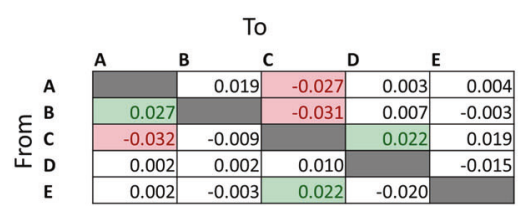

MDD

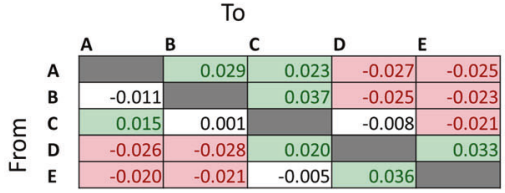

\section{Control vs rMDD}

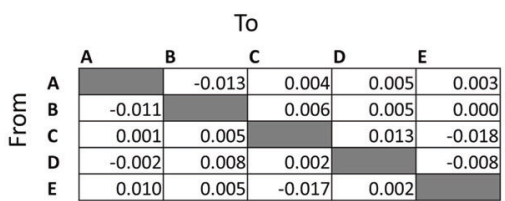

rMDD

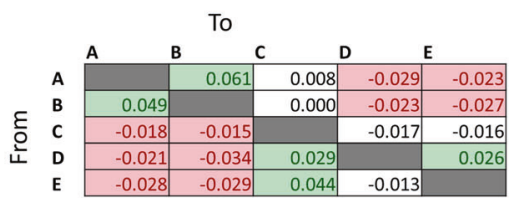

rMDD vs MDD

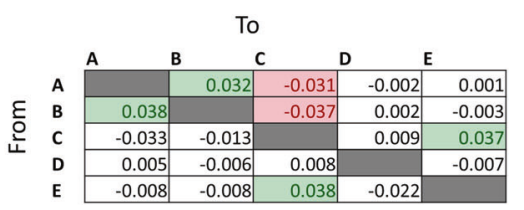

Fig. 4 Microstate transition probabilities are non-random and are abnormal in MDD. a Matrix showing the observed transition probabilities given the known distribution of microstate labels minus the expected transition probabilities. Positive values indicate more transitions than would be expected by chance, negative values indicate fewer transitions. Colored boxes and text indicate statistically significant differences as calculated by Bonferroni-adjusted t-tests. b Comparisons of observed minus expected transitions between HC, MDD, and rMDD groups. Positive values indicate that there are more transitions in the first listed group compared to the second, red values indicate that there are more transitions in the second listed group compared to the first. Colored boxes and text indicate statistically significant differences as calculated by ANOVAs with post-hoc HSD test $(p<0.05)$.

Transitions between microstates are non-random and some transitions are selectively altered in MDD

In order to study microstate syntax, we used a Markov analysis in which we conditioned the proportion of microstate transitions between each pair of states by the proportion of such transitions that would be expected by a random process given the distribution of microstate labels for each recording [21]. We found evidence that specific transitions that were clearly preferred and others that were relatively disfavored relative to what would be expected from a random process (Fig. 4a). For all groups, most observed transition probabilities were statistically significantly different from the expected transition probabilities (Fig. 4a, paired $t$-tests, with $\mathrm{p}_{\text {Bonferroni }}<0.05$ ). Overall, there was a qualitatively similar pattern of transition results across the three groups. In general, microstates $A$ and $B$ transitioned to microstates $D$ and $E$ less often than would be expected by the distribution of labels, and vice versa. Microstates $D$ and $\mathrm{E}$ preferentially transitioned to microstate $\mathrm{C}$.

We next tested whether there were differences in these distribution-adjusted microstate transitions among the HC, MDD, and rMDD groups. For each type of transition, we performed an ANOVA across groups. The $p$-values from the ANOVAs were Bonferroni-corrected for the 20 transitions. Post-hoc tests showed several differences between the $\mathrm{HC}$ and MDD groups and between the rMDD and MDD groups, but no significant differences between the HC and rMDD groups (Fig. 4b). Most of these transition differences involved microstate $C$. Relative to both the $\mathrm{HC}$ and $\mathrm{rMDD}$ group, the MDD group had more transitions from microstate $A$ and $B$ to $C$, and more from microstate $E$ to $C$. In addition, relative to both the $\mathrm{HC}$ and $\mathrm{rMDD}$ groups, the MDD group had fewer transitions from microstate $B$ to microstate $A$.

Finally, we assessed the association between microstate $A$ to $C$, $B$ to $C$, and $E$ to $C$ transition probabilities and BDI-II scores. Within MDD patients with BDI-II scores greater than 9, we found a positive correlation between microstate $B$ to $C$ transitions and $B D I-$ II scores (Spearman's rho $=0.31, \mathrm{p}_{\text {Bonferroni }}=0.043$ ). There were no statistically significant correlations between medication status and any transition probability (all unadjusted $p>0.13$ ).

\section{DISCUSSION}

We used microstate analysis to characterize resting-state EEG in $H C, M D D$, and rMDD. For all groups, five microstates best fit the data. These sets of microstates included four widely reported canonical microstates A-D. The additional microstate $E$ has also been reported in previous studies $[8,14]$. The cortical correlates of these microstates overlap with multiple fMRI resting-state networks. Because resting-state networks have previously been found to be abnormal in MDD, we hypothesized that there would be differences in microstates between MDD patients and controls [2]. We found that microstate $D$ proportion and occurrence were decreased in both the MDD and rMDD compared to the HC group and microstate $D$ duration was decreased in the MDD group. Moreover, microstate $A$ occurrence was increased in MDD relative to $\mathrm{HC}$ and $\mathrm{rMDD}$. Microstate transitions, particularly involving microstate $C$, were different in MDD compared to HC and rMDD. The effects were unrelated to age, education, or medication.

Microstates as a tool to probe brain function

Microstate analyses provide information about the activity of largescale neural assemblies [11], with microstate topography being determined by the spatial distribution of underlying assemblies. Microstate duration is a measure of how long the brain maintains each semi-stable state, and is influenced by cortical and subcortical brain function [10,33]. The cortical generators of EEG microstates have been related to fMRI resting-state networks [34, 35]. The details of this relationship are controversial, with some proposing that there are clear, one-to-one mappings between microstates and restingstate networks [34] and others arguing that the cortical generators of microstates overlap with, but are distinct from, resting-state networks or that microstate analysis shows that fMRI-defined resting-state networks can be subdivided into functionally distinct sub-networks $[9,10,26]$. Our results suggest that microstates do not directly correlate with fMRI resting-state networks and instead provide unique information about the activity of neural assemblies. Accordingly, microstate analysis provides new information about the brain and additional insight into the pathophysiology of psychiatric diseases.

\section{Microstates and MDD}

There is limited research capitalizing on microstate analysis to investigate affective disorders, and no previous study has specifically focused on MDD or included an rMDD sample. Results have been inconsistent, with reports that (1) the longest duration microstates were shorter in a mixed depressive cohort when compared to controls, but that overall mean duration was equivalent [12]; (2) microstates in the beta frequency band showed no difference in overall mean microstate duration between MDD and HC [36]; (3) electroconvulsive therapy- 
induced increased duration of microstate $A$ and decreased occurrence of microstates B, C, and D in patients with treatment-resistant depression, with effects most pronounced in treatment responders [37]; (4) a small, mixed cohort of patients with active depressive disorders did not differ from controls in microstate topography, occurrence, or duration but microstate $A$ occurrence was positively correlated with depression symptoms [14]; and (5) a mixed sample of patients with mood and anxiety disorders did not differ from controls in microstate topography but was characterized by decreased transitions from microstate $B$ to $D$ and increased transitions from $A$ to $D$ and $B$ to $C$ [13].

The current work is the largest microstate study of patients with affective disorders to date that focuses specifically on unipolar MDD. Our findings partially agree with prior results. We report similar microstate topographies in the HC, MDD, and rMDD groups suggesting that the cortical generators of the microstate maps are largely preserved. Unlike prior reports, a clear decrease in microstate $D$ (in proportion, duration, and occurrence) emerged. In agreement with Al Zoubi et al., we note increased transitions from microstate B to $C$ [13]. However, they described other transitions that are not abnormal here and we report disorder-related transition differences that they did not detect. These discrepancies may result from differences in data acquisition and/or analysis. In particular, they note multiple transitions involving canonical microstate $D$. While we did identify microstate $D$, a topography resembling our microstate $E$ has been previously labeled microstate $D$ in other studies $[38,39]$. This suggests that Al Zoubi et al.'s microstate D may be the sum of our microstates $D$ and $E$. Decreased microstate $D$ has also been reported in psychotic disorders suggesting a common pathophysiological process across affective and psychotic disorders [40]. This process may be related to microstate D's proposed association with cognitive control and ability to attend to environmental stimuli and which may manifest as rumination in depression or thought disorder in psychosis [26]. Fitting prior reports, we showed that microstates $A$ and $B$ preferentially transition between each other [17]. This pattern is also disrupted in psychotic disorders, highlighting a potential shared neural abnormality between affective and psychotic disorders [17].

Microstate $C$ has been related to activity in the precuneus in the current and previous work [8]. The association between precuneus activity and multiple different microstates has led to the hypothesis that the precuneus links multiple functional networks [8]. We note that the altered transition probabilities mostly involve microstate $C$. This suggests that there may be dysfunction in the ability of the precuneus to appropriately sequence transitions between large scale networks in patients with MDD. Increased transitions from microstate $A$ and $B$ to microstate $C$ implicate increased connectivity from components of the default mode network and fronto-parietal network to the precuneus. This is in agreement with past work showing increased default mode network and fronto-parietal network connectivity in MDD (for review see [3]). However, we found that only some components of these networks have increased connectivity. Therefore, different components within a given resting-state network have distinct connectional abnormalities in MDD. We note that the changes in microstate transitions are not driven by an increase in noise, rather, there are distinct, statistically significant deviations from random that exist uniquely in the MDD data. There may be abnormal processes that shape microstate transitions in patients with MDD and that remission is accompanied by cessation of, or compensation for, these processes. Previous work has demonstrated anatomical abnormalities in the precuneus in patients with both MDD and rMDD [41,42]. Therefore, the recovery of normal microstate transition probabilities that we observed in rMDD group is likely driven by compensatory processes occurring outside the precuneus.

In sum, previous functional and structural MRI work suggests that MDD is a disconnection syndrome $[2,43]$. Our findings agree with, and expand on, these prior studies. Past work has demonstrated hyperconnectivity between anterior and posterior components of the default mode network in MDD [2]. Microstates A and B include many of the anterior and posterior components of this network, and we found that transitions between these two microstates are increased in MDD. Alternatively, we note that decreased transitions from microstate $C$ to microstate $A$ and $B$, as well as the increased transitions from microstate $C$ to microstate $E$, have no clear correlate in the fMRI literature. Our findings suggest that MDD is associated with dysconnectivity. This may be due to subcortical structures, which are also known to be disrupted in MDD [44]. While subcortical activity may contribute to the EEG, the relationship between this component of EEG and microstates is unclear [45]. Remission of MDD may require large-scale compensatory changes in brain connectivity, even in regions that are not directly implicated in MDD. Collectively, our findings provide evidence that EEG microstate analysis provides novel information about brain function.

\section{Limitations}

Several limitations should be considered. First, understanding the cortical correlates of EEG microstates relies on source modeling techniques, which have, at best, limited spatial resolution. These methods rely on multiple biophysical assumptions and simplifications that are still undergoing refinement [46]. Second, groups using various analysis techniques and acquisition setups have reported sets of EEG microstates with different topographies and longer durations $[11,13,14]$. However, the majority of studies have reported at least the four canonical microstates that we also found. Third, although the inclusion of a rMDD group enabled inferences regarding clinical state, a longitudinal approach would provide additional certainty that microstate syntax differences are truly state-markers of MDD.

\section{FUNDING AND DISCLOSURE}

This work was supported by National Institute of Mental Health Grants K23 MH118565 (to MM), R01 MH095809, R01 MH101521 and R37 MH068376 (to DAP), and Carol Silverstein and Jill Gotlieb of The William Rosenberg Family Foundation). AEW was partially supported by fellowships from the Andrew P. Merrill Memorial Fund, the Brain and Behavior Research Foundation, and the National Health and Medical Research Council of Australia (Grant No. APP1110773). MDS is partially supported by an award funded by the Phyllis and Jerome Lyle Rappaport Foundation. The content is solely the responsibility of the authors and does not necessarily represent the official views of the National Institutes of Health. The authors are grateful to Nancy Hall-Brooks, Laurie Scott, Madeline Alexander, and David Crowley for their contributions to clinical assessments. Over the past 3 years, DAP has received consulting fees from Akili Interactive Labs, BlackThorn Therapeutics, Boehringer Ingelheim, Compass Pathway, Otsuka Pharmaceuticals, Posit Science, and Takeda Pharmaceuticals, as well as stock options from BlackThorn Therapeutics and one honorarium from Alkermes for activities unrelated to the current work. Over the past 3 years, MDS has received consulting fees from Vorso Corp., for activities unrelated to the current work. No funding from these entities was used to support the current work, and all views expressed are solely those of the authors. All other authors report no biomedical financial interests.

\section{AUTHOR CONTRIBUTIONS}

MM: performed all analyses and drafted the first version of the paper. AEW: contributed to data analyses and interpretation, and provided revision and important intellectual content to the manuscript. SD, MLI, AR and MB: contributed to data collection and processing. MS: contributed to data interpretation, and provided revision and important intellectual content to the manuscript. DAP: designed the studies that contributed data, contributed to data interpretation, provided revision 
and important intellectual content to the manuscript, and provided funding. All authors approved the final version of the manuscript. MM and DAP take responsibility for the accuracy and integrity of any part of the work.

\section{ADDITIONAL INFORMATION}

Publisher's note Springer Nature remains neutral with regard to jurisdictional claims in published maps and institutional affiliations.

\section{REFERENCES}

1. Baxter AJ, Scott KM, Ferrari AJ, Norman RE, Vos T, Whiteford HA. Challenging the myth of an 'epidemic' of common mental disorders: trends in the global prevalence of anxiety and depression between 1990 and 2019. Depress Anxiety. 2014;31:506-516.

2. Kaiser RH, Andrews-Hanna JR, Wager TD, Pizzagalli DA. Large-scale network dysfunction in major depressive disorder a meta-analysis of resting-state functional connectivity. JAMA Psychiatry. 2015;72:603-611.

3. Whitton AE, Deccy S, Ironside ML, Kumar P, Beltzer M, Pizzagalli DA. EEG source functional connectivity reveals abnormal high-frequency communication among large-scale functinoal networks in depression. Biol Psychiatry Cogn Neurosci Neuroimaging. 2018;3:50-58.

4. Lehmann D. Brain electric microstates and cognition: the atoms of thought. In: John ER, editor. Mach. Mind, Boston: Birkhäuser; 1990. p. 209-224.

5. Strik WK, Lehmann D. Data-determined window size and space-oriented segmentation of spontaneous EEG map series. Electroencephalogr Clin Neurophysiol 1993;87:169-174.

6. Lehmann D, Michel CM. Clinical Neurophysiology EEG-defined functional microstates as basic building blocks of mental processes. Clin Neurophysiol 2011;122:1073-1074.

7. Michel CM, Koenig T. EEG microstates as a tool for studying the temporal dynamics of whole-brain neuronal networks: A review. Neuroimage 2018;180:577-593.

8. Custo A, Van De Ville D, Wells WM, Tomescu MI, Brunet D, Michel CM. Electroencephalographic resting-state networks: source localization of microstates. Brain Connect 2017;7:671-682.

9. Yuan H, Zotev V, Phillips R, Drevets WC, Bodurka J. Spatiotemporal dynamics of the brain at rest - Exploring EEG microstates as electrophysiological signatures of BOLD resting state networks. Neuroimage 2012;60:2062-2072.

10. Milz P, Pascual-Marqui RD, Achermann P, Kochi K, Faber PL. The EEG microstate topography is predominantly determined by intracortical sources in the alpha band. Neuroimage 2017;162:353-361.

11. Khanna A., Pascual-Leone A., Michel C. M., Farzan F. Microstates in resting-state EEG: current status and future directions. Neurosci Beiobhav Rev. 2015;49:105-113.

12. Strik WK, Dierks T, Becker T, Lehmann D. Larger topographical variance and decreased duration of brain electric microstates in depression. J Neural Transm 1995;99:213-222.

13. Al Zoubi O, Mayeli A, Tsuchiyagaito A, Misaki M, Zotev V. EEG microstates temporal dynamics differentiate individuals with mood and anxiety disorders from healthy subjects. Front Hum Neurosci 2019;13:1-10.

14. Damborska A, Tomescu MI, Honzirkova E, Bartecek R, Horinkova J, Fedorova S, et al. EEG resting-state large-scale brain network dynamics are related to depressive symptoms. Front Psychiatry 2019;10:548.

15. First MB, Spitzer RL, Gibbon M,Williams JB. Structured Clinical Interview for DSMIV-TR Axis I disorders, research version, patient edition (pp. 94-1) (SCID-I/P) Biometric Research, New York State Psychiatric Institute, New York, NY 2002.

16. Beck AT, Steer RA, Brown GK. Beck Depression Inventory Manual. 2. San Antonio: The Psychological Corporation; 1996.

17. Murphy M, Stickgold R, Öngür D. Electroencephalogram microstate abnormalities in early-course psychosis. Biol Psychiatry Cogn Neurosci Neuroimaging. 2020;5:35-44.

18. Makeig S, Bell AJ, Jung T-P, Sejnowski TJ. Independent component analysis of electroencephalographic data. Adv Neural Inf Process Sys. 1996;8:145-151.

19. Perrin F, Pernier J, Bertrand O, Echallier JF. Spherical splines for scalp potential and current density mapping. Electroencephalogr Clin Neurophysiol 1989;72:184-187.
20. Murray MM, Brunet D, Michel CM. Topographic ERP analyses: a step-by-step tutorial review. Brain Topogr 2008;20:249-264.

21. Brunet D, Murray MM, Michel CM. Spatiotemporal analysis of multichannel EEG: CARTOOL. Comput Intell Neurosci. 2011;2011:1-15.

22. Michel CM, Brunet D. EEG Source Imaging: A Practical Review of the Analysis Steps. Front. Neurol. 2019:10:325. https://doi.org/10.3389/fneur.2019.00325

23. Charrad M, Ghazzali N, Boiteau V, Niknafs A. NbClust: An R Package for Determining the Relevant Number of Clusters in a Data Set. J Stat Softw. [Online], 61.6 2014:1-36.

24. Milligan GW, Cooper MC. An examination of procedures for determing the number of clusters in a data set. Psychometrika 1985;50:159-179.

25. Krzanowski WJ, Lai YT. A criterion for determining the number of groups in a data set using sum-of-squares clustering. Biometrics 1988;44:23-34.

26. Brechet L, Brunet D, Birot G, Gruetter R, Michel CM, Jorge J. Capturing the spatiotemporal dynamics of self-generated, task-initiated thoughts with EEG and fMRI. Neuroimage 2019;194:82-92.

27. Pascual-Marqui RD, Michel CM, Lehmann D. Segmentation of brain electrical activity into microstates; model estimation and validation. IEEE Trans Biomed Eng 1995;42:658-665

28. Gärtner M, Brodbeck V, Laufs H, Schneider G. A stochastic model for EEG microstate sequence analysis. Neuroimage 2015;104:199-208.

29. Tadel F, Baillet S, Mosher JC, Pantazis D, Leahy RM. Brainstorm: a user-friendly application for MEG/EEG analysis. Comput Intell Neurosci 2011;2011:1-13.

30. Fonov V, Evans AC, Botteron K, Almli CR, Mckinstry RC, Collins DL, et al. Unbiased average age-appropriate atlases for pediatric studies. Neuroimage 2011;54:313-327.

31. Pascual-Marqui RD. Standardized low-resolution brain electromagnetic tomography (sLORETA): technical details. Methods Find Exp Clin Pharmacol 2002;24 (Suppl D):5-12.

32. Koenig T, Prichep L, Lehmann D, Sosa PV, Braeker E, Kleinlogel H, et al. Millisecond by millisecond, year by year: normative EEG microstates and developmental stages. Neuroimage 2002;16:41-48.

33. Hughes SW, Crunelli V. Thalamic mechanisms of EEG alpha rhythms and their pathological implications. Neuroscientist 2005;11:357-372.

34. Britz J, Van De Ville D, Michel CM. BOLD correlates of EEG topography reveal rapid resting-state network dynamics. Neuroimage 2010;52:1162-1170.

35. Musso F, Brinkmeyer J, Mobascher A, Warbrick T, Winterer G. Spontaneous brain activity and EEG microstates. A novel EEG/fMRI analysis approach to explore resting-state networks. Neuroimage 2010;52:1149-1161.

36. Ihl R, Brinkmeyer J. Differential diagnosis of aging, dementia of the Alzheimer type and depression with EEG-segmentation. Dement Geriatr Cogn Disord 1999;10:64-69.

37. Atluri S, Wong W, Moreno S, Blumberger DM, Daskalakis ZJ, Farzan F. Selective modulation of brain network dynamics by seizure therapy in treatment-resistant depression. Neuroimage (Amst). 2018;20:1176-1190.

38. Brodbeck V, Kuhn A, von Wegner F, Morzelewski A, Tagliazucchi E, Borisov S, et al. EEG microstates of wakefulness and NREM sleep. Neuroimage 2012; 62:2129-2139.

39. Seitzman BA, Abell M, Bartley SC, Erickson MA, Bolbecker AR, Hetrick WP. Cognitive manipulation of brain electric microstates. Neuroimage 2017 146:533-543.

40. Rieger K, Hernandez LD, Baenninger A, Koenig T. 15 years of microstate research in schizophrenia - Where are we? A meta-analysis. Front Psychiatry 2016;7:1-7.

41. Zhang Y, Yu C, Zhou Y, Li K, Li C, Jiang T. Decreased gyrification in major depressive disorder. Neuroreport 2009;20:378-380.

42. Zhang S, Li, C-SR. Functional connectivity mapping of the human precuneus by resting state fMRI. Neuroimage 2013:59:3548-3562.

43. Liao $Y$, Huang $X$, Wu Q, Yang $C$, Kuang $W$, Du $M$, et al. Is depression a disconnection syndrome? Meta- analysis of diffusion tensor imaging studies in patients with MDD. J Psychiatry Neurosci 2013;38:49-56.

44. Nugent AC, Davis RM, Zarate CA Jr, Drevets WC. Reduced thalamic volumes in major depressive disorder. Psychiatry Res 2014;213:179-185.

45. Seeber M, Cantonas LM, Hoevels M, Sesia T, Visser-Vandewalle V, Michel CM. Subcortical electrophysiological activity is detectable with high-density EEG source imaging. Nat Commun 2019;10:1-7.

46. Nunez PL and Srinivasan R. Electric fields of the brain: the neurophysics of EEG. Oxford University Press. 2006. 